\title{
A practical localization solution for wireless sensor networks deployed in linear topography
}

\author{
Kui Zhang \#, Peng Guo *, Nirvana Meratnia \#, Paul J.M. Havinga \# \\ \# Pervasive Systems, University of Twente \\ \{K. Zhang, N.Meratnia, P.J.M.Havinga\}@ewi.utwente.nl \\ * Department of Electronic and Engineering \\ Huazhong University of Science and Technology \\ *guopeng@hust.edu.cn
}

\begin{abstract}
In this paper, we propose a practical range-free localization solution for wireless sensor networks (WSNs). Different from existing localization approaches, the proposed solution is specially designed for an ultra sparse mobile WSNs deployed in coal mine tunnels with linear topography. To obtain more reference information requited for localization with limited anchor nodes, the proposed solution sets asymmetrical transmission range for the sensor nodes and makes best use of a priori knowledge about the location of the anchor nodes. Numerous real measurements and real localization experiments were made in a coal mine tunnel in the ShanDong province, China. The experimental results show that our proposed solution achieves a high localization accuracy with an error less than $13 \%$ of the transmission range of the anchor nodes, while, the traditional range-free solution, i.e., Ad-Hoc Positioning System, has an error about $44 \%$ of the transmission range under the same conditions.
\end{abstract}

Index Terms-Wireless sensor networks, linear topology, localization, range-free, asymmetrical transmission range.

\section{INTRODUCTION}

Wireless sensor networks (WSNs) have been widely used in various applications. In recent years, many WSNs have been deployed in coal mines to monitor the environment and help search and rescue operation. In these operations, knowing miners' positions could be highly beneficial. Global Positioning System (GPS) is a well-known and widely used localization system, however, the GPS signal cannot be received underground. Therefore, localization based on wireless sensor networks has received much attention. As coal mine tunnels are usually long (tens of kilometers) and narrow (several meters), the WSN deployed in the tunnels has a linear topology. The main issue of localization here is to obtain the distance between a miner and entrances of the tunnel.

Many localization algorithms for WSN have been proposed, which in general can be classified as range-based and rangefree. The range-based algorithms usually use the distance measured based on the packet arrival time [1] and the angle measured from the angle of arrival signals [2] to calculate the location. Although accuracy of range-based localizations is often high, they have high hardware and energy expenditure. It is hard to envision cheap, simple, and reliable WSN devices that can be used for range-based solutions. Some other rangebased solutions use the signal strength to estimate the pointto-point distance [3], [4]. These approaches can be easily implemented but the signal strength has a very dynamic range, which could lead to a low localization accuracy.

Range-free localization algorithms such as [5], [6], [7], [8], [9], which do not require the availability and validity of the range information, have been pursued as cost-effective alternatives for expensive range-based schemes. Most of these solutions are based on the methodology proposed in the ad-hoc positioning system (APS) [5]. The key idea here is to place small fraction of anchors with known coordinates across the network. Location of the sensor nodes is obtained from the estimated distance to multiple anchors. The location errors can be masked by features such as node redundancy and data aggregation [10], [11]. However in sparse networks, the performance and accuracy of range-free localization techniques will greatly deteriorate and the location error will increase to such an extent to be more than $100 \%$ of the transmission range [5].

It is not expected to deploy a large number of sensor nodes in the coal mine tunnels simply because their deployment, management, and maintenance are difficult, costly, and labourintensive. Therefore, low node density in long and narrow coal mine tunnels is a real challenge for localization. Moreover, the nodes attached to miners move around frequently. It is also important to be able to perform localization even if a node (miner) does not have any neighbor. In addition it has been shown that in a topography like a tunnel, the radio signals can be transmitted much further than in a common indoor environment. In this case, many range-free solutions which locate a destination node simply just based on whether it is in an anchor node's communication range, have very low accuracy. All these make localization in coal mine tunnels to be very different from other usual scenarios.

To this end, there are only a few localization solutions applicable to the coal mine tunnels, however, they are not so effective. Most of them use radio-frequency identification (RFID), in which few RFID readers are deployed in the tunnels with a gap of several hundred meters. When the miners who 
carry RFID tags pass a RFID reader, it would record the miner according to the IDs programmed in the tags. Localization accuracy of this technique is about hundred meters (distance between RFID readers), which is too low to be able to effectively help the search and rescue operation.

In this paper, we propose a practical range-free localization for ultra low density linear wireless sensor networks to locate miners in the coal mine tunnels. As there are only limited anchor nodes, we use asymmetrical transmission range to get more reference information which will be used to estimate the location of the mobile sensor nodes carried by miners. This solution is density independent and can even work well with presence of only one miner in the tunnel. We have made several experiments in a real coal mine with limited anchor nodes and compared the performance with the Ad-Hoc Positioning System presented in [5].

\section{Communication test}

\section{A. Communication test in a coal mine tunnel}

It is well-known that the transmission range varies when the transmission power changes. But the transmission range in different working environments even varies with the same power level. We have performed many measurements to find the relationship between the transmission range and power level in various environments for the most widely used radio chip $C C 2420$. In our experiments in a coal mine in the ShanDong province in China, the sensor nodes with $C C 2420$ radio transmit packets every $5 \mathrm{~ms}$. We measured the packet receiving ratio in duration of $1 \mathrm{~s}$ and made 200 measurements at every 10 meters with four different transmission power levels. Figure 1 shows the average value of the packet receiving ratio as well as the maximum and minimum value for 200 tests.

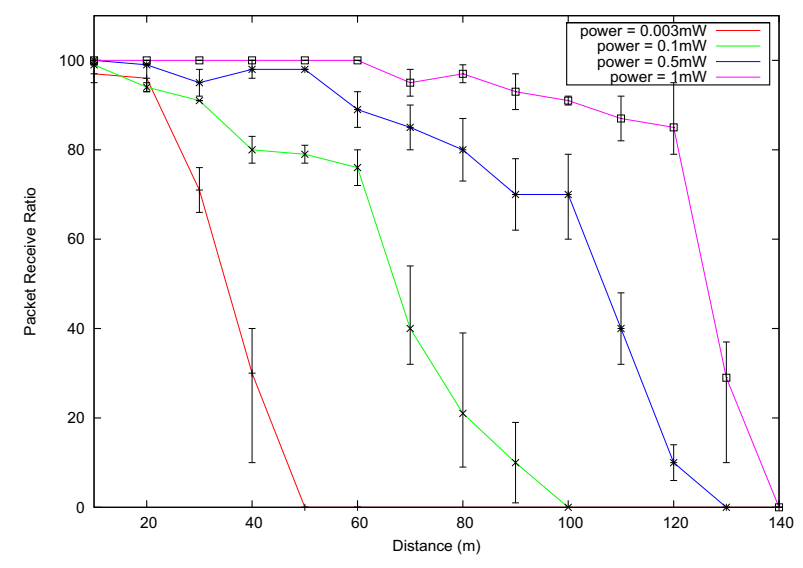

Fig. 1. Average value of the packet receiving ratio as well as the maximum and minimum value for 200 tests performed in the coal mine tunnel

It can be seen from Figure 1 that when transmission power is $1 \mathrm{~mW}$, the transmission range of the sensor node is about 120 meters. This is much longer than the transmission range of node in indoor environments. On the other hand, when transmission power is $3 \mu W$, the transmission range is about 30 meters. Considering the fact that the wireless communication is unstable, the maximal transmission range is irregular and dynamic in practice so we redefine the transmission range as the distance where the packet receiving ratio can always be more than $80 \%$. Moreover the critical distances in different directions are different, thus we choose the smaller one as the transmission range of the node.

\section{B. Deployment}

Based on the characteristics of the transmission in the coal mine described above, we propose a practical localization for ultra sparse mobile WSNs deployed in the coal mines. We deploy a number of anchor nodes in such a way to cover the whole coal mine tunnel as shown in Figure 2.

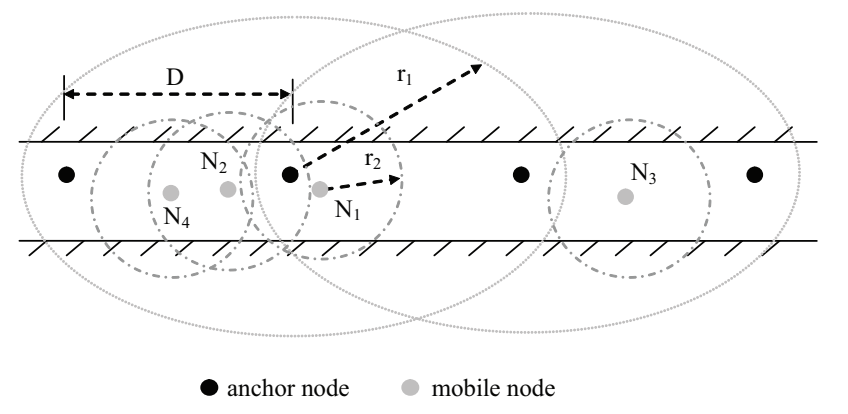

Fig. 2. Our coal mine tunnel deployment

The black nodes in Figure 2 are anchor nodes and the gray nodes are the mobile nodes carried by the miners. Distance between adjacent anchor nodes, deployed along the tunnel, are equal. Location of the anchor nodes will be made known to the whole network through broadcasting in the localization phase. All the sensor nodes use the radio chip $\mathrm{CC} 2420$ at $2.4 \mathrm{GHz}$ frequency. The anchor nodes communicate with the highest power level of $1 \mathrm{~mW}$ which results in a transmission range of $r_{1}$ in the coal mine. In order to increase localization accuracy, we set the gap between anchor nodes to be $D=r_{1} / 112.5 \%$ (i.e. $r_{1}=112.5 \% * D$ ), meanwhile the mobile nodes work with a small power level which results in a transmission range of about $r_{2}=37.5 \% D \approx 33.3 \% * r_{1}$.

As the proposed algorithm is range-free, we only use the connection information in the network to calculate the location of the mobile sensor nodes. After the mobile node affirms its location, it sends the localization result to the anchor nodes with maximum power. Those anchor nodes receiving the location information will forward the message to the control center placed outside the coal mine, so location of the miners in the coal mine can be known.

\section{LINEAR LOCALIZATION IN ULTRA SPARSE MOBILE WSNS}

In our algorithm, we basically use asymmetrical transmission range and anchor nodes' location to estimate the location of the mobile nodes. 


\section{A. Analysis of asymmetrical transmission range}

We first define the following two terminologies:

- Full reference link: If a mobile node $M$ and an anchor node $A$ are both in the transmission range of each other, then the mobile node $M$ has a full reference link.

- Half reference link: If a mobile node $M$ is in the transmission range of an anchor node $A$ but $A$ is not in the transmission range of $M$, then the mobile node $M$ has a half reference link.

Thus we can define states of the mobile nodes according to their reference links. Table I presents the reference link information for the four mobile nodes shown in Figure 2.

\begin{tabular}{|c|c|c|}
\hline Node & Full reference link & Half reference link \\
\hline$N_{1}$ & 1 & 2 \\
\hline$N_{2}$ & 1 & 1 \\
\hline$N_{3}$ & 0 & 2 \\
\hline$N_{4}$ & 0 & 2 \\
\hline
\end{tabular}

TABLE I

REFERENCE LINK OF MOBILE SENSOR NODES

The relationship between the transmission range and gap between anchors in the deployment is expressed as $r_{1}=$ $112.5 \% * D$. Thus, every mobile sensor node can only have the following states:

- One full reference link and two half reference links, when the mobile sensor node is in the range $R A_{1}=$ $[0,12.5 \% D] \cup[87.5 \% D, D]$.

- One full reference link and one half reference link, when the mobile sensor node is in the range $R A_{2}=$ $(12.5 \% D, 37.5 \% D] \cup[62.5 \% D, 87.5 \% D)$.

- Two half reference links, when the mobile sensor node is in the range $R A_{3}=(37.5 \% D, 62.5 \% D)$.

The reference links of a mobile node moving around in an $[0, D]$ area in the tunnel can be computed using the following reference link function $f_{\text {ref-links }}(x)$ :

$$
f_{\text {ref-links }}(x)= \begin{cases}F+2 H, & x \in R A_{1}, \\ F+H, & x \in R A_{2}, \\ 2 H, & x \in R A_{3},\end{cases}
$$

, in which function $F$ denotes full reference links and $H$ denotes half reference links. The reference function $f_{\text {ref-links }}(x)$ is the core technique used in our algorithm. As the function shows, every mobile sensor node in the tunnel can only have the three reference link states illustrated in Figure 3.

Furthermore, the connection between two neighboring mobile nodes can be used to improve the localization accuracy. When two neighboring mobile nodes have obtained their location ranges according to the full reference links and half reference links, they can further reduce their location ranges according to the optional reference link, since their transmission range is no more than $37.5 \% \mathrm{D}$, i.e., the distance between the two nodes is no more than $37.5 \% \mathrm{D}$.
However, not all the connections between the mobile nodes can be used to improve the localization accuracy. When two neighboring nodes are in the same location range or adjacent location ranges, the connection between them can not povide any extra reference of location; when two neighoring nodes are in two disjunct location ranges, the connection between them can provide some extra reference.

Hence, for convenience, we call the connection between two mobile nodes in two disjunct location ranges as optional reference link.

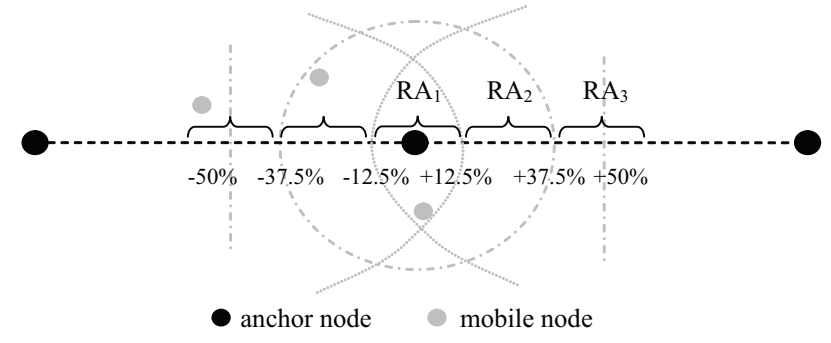

Fig. 3. Analysis of communication range

\section{B. Description of the solution}

After analyzing the asymmetrical communication ranges, described above, we introduce the process of the proposed localization solution:

1) Get the states of reference links by just listening to the beacons from anchor nodes without sending packets back. Here the reference links are the links with good quality via which the receiving ratio is better than $90 \%$. As the analysis in the above section shows, the mobile nodes can have two different states.

2) In presence of three anchor nodes with locations $x_{1}, x_{2}$ and $x_{3}\left(x_{1}<x_{2}<x_{3}\right)$, a rough estimation of the mobile node's location range can be made $\left(x_{2}-12.5 \% D, x_{2}+\right.$ $12.5 \% D)$. In this case step 4 is executed. In presence of only two anchor nodes with locations $x_{1}$ and $x_{2}$, step 3 is executed.

3) The mobile node sends $E C H O$ packets to the two anchor nodes that it has heard in step 2 and then checks whether there is an $A C K \_E C H O$ packet from any anchor nodes. If there is one with location $x_{1}$, it calculates its location range based on $\left(x_{1}+12.5 \% D, x_{1}+37.5 \% D\right)$. If location of the answering anchor node is $x_{2}$, the mobile node calculates its location range based on $\left(x_{2}-37.5 \% \mathrm{D}\right.$, $\left.x_{2}-12.5 \% D\right)$. If there is no answering anchor node, the mobile node calculates its location range based on $\left(x_{1}+\right.$ $\left.37.5 \% D, x_{2}-37.5 \% D\right)$. After that, step 4 is executed.

4) The mobile node which has already got its location range $\left(l_{1}, l_{1}+25 \% D\right)$, checks whether there is any optional reference link by broadcasting $M O B I L E_{-} E C H O$ packet. If there is one optional reference link with another moile node whose location range is $\left(l_{2}, l_{2}+25 \% D\right)$ (suppose $l_{2}>l_{1}$ ), the former mobile node will affirm its location as $l_{1}+18.75 \% D$ and the latter will affirm its location as $l_{2}+6.25 \% D$. 
5) The mobile node sends its calculated location to the nearest anchor node with maximal transmission power.

Note that, when a mobile node detects multiple optional reference links, it can not further improve the location accuracy if one of them has already been used. This is because the corresponding mobile nodes for the multiple optional reference links must be in the same location range with the transmission range of the mobile nodes being restricted. Thus if there are several optional reference links, just choose one of them and ignore the others.

A detailed illustration of the proposed localization solution is shown in Figure 4.

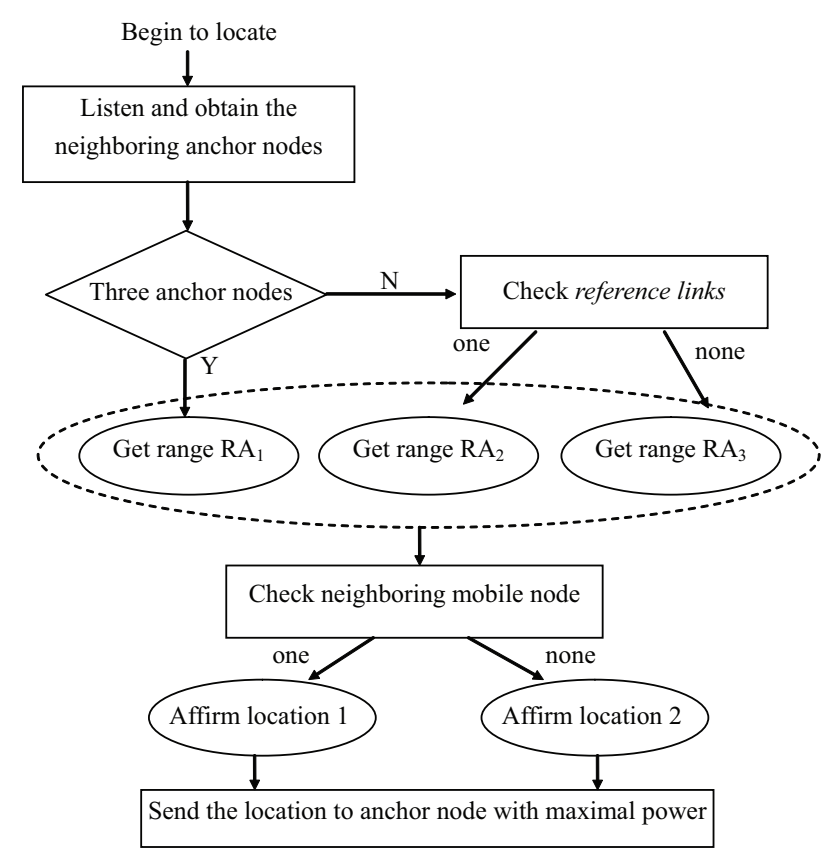

Fig. 4. Process of the localization solution

\section{Performance analysis of the proposed solution}

According to the solution, the real location of the mobile nodes are in the range $\left(l_{x}, l_{x}+25 \% D\right)$ and we estimate them as $l_{x}+12.5 \% D$. That is to say the maximum location error is less than $12.5 \% D \approx 11 \% r_{1}$.

Furthermore, if there is optional reference link as shown in Figure 5, we estimate the location as $l_{x}+18.75 \% D$ accroding to the proposed solution. Hence the location error is no more than $6.25 \% D \approx 5.5 \% r_{1}$.

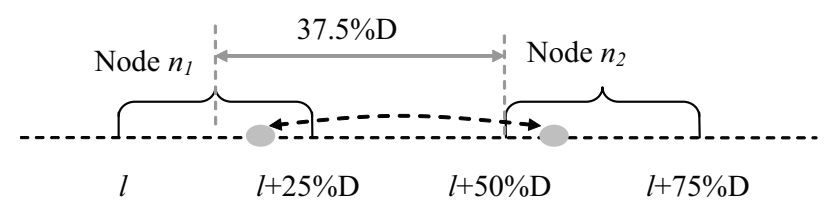

Fig. 5. An Example of Optional Reference Link

\section{EXPERIMENTS}

To evaluate our proposed localization solution, we made some experiments in a main tunnel of a real coal mine without considering the branch cases in ShanDong province, China. There are four anchor nodes, being 100 meters apart, hang from a wire rope as shown in Figure 6 and their transmission power is $1 \mathrm{~mW}$. A mobile node with transmission power of $30 \mu W$ was carried by one of our teammates who moved around in this area. In this experiment, the mobile nodes calculate their locations every $120 \mathrm{~ms}$ which has little influence on the accuracy because the normal speed of people is only $2 \mathrm{~m} / \mathrm{s}$. We repeated our experiments 10 times at every 10 meters between the two middle anchor nodes. All the nodes sent beacon packets every $20 \mathrm{~ms}$ with a TDMA communication scheme. If the anchor node receives $E C H O$ packet from the mobile nodes, it then sends an $A C K_{-} E C H O$ packet back; if the mobile sensor node which has already got its location receives $M O B I L E_{-} E C H O$ packet from other mobile nodes, it then sends the $A C K_{-}$MOBILE_ECHO packet with its location back. The experiment results are shown in Figure 7.

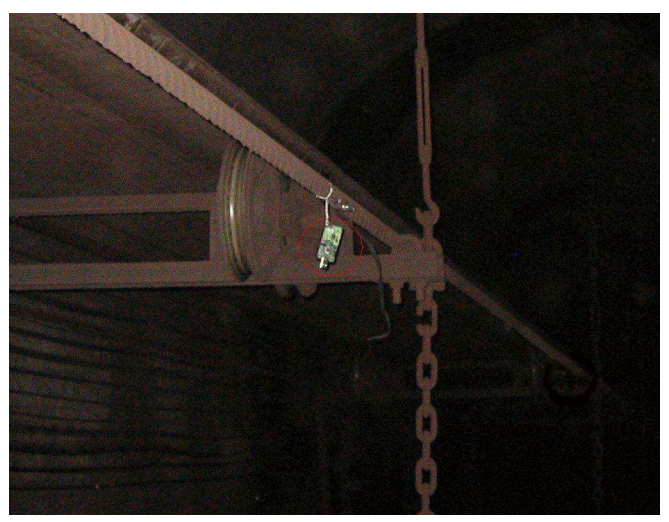

Fig. 6. Experimental setup in the coal mine

From Figure 7, it can be seen that the maximal location error is 15 meters which is just $13 \%$ of the transmission range of the anchor nodes. That is to say that the error is little larger than the maximum error in theory which is $11 \%$ of the transmission range. This is because the three location ranges are not even in practice. There is very little error at the locations of 0 meters, 50 meters and 100 meters. For comparison, we also present location error of the APS measured in the same environment, shown as a line. As APS estimates the location of a mobile node just according to the common range of anchor nodes where the mobile node is, the location of the mobile node is always at 50 meters. This results in a large error as much as $44 \%$ of the transmission range when the mobile node is at 0 meter or 100 meters.

\section{CONCLUSION}

The linear topology with tens of kilometers length, availability of a few anchor nodes, ultra sparse characteristics of mobile nodes (miners) and large transmission range of sensor nodes, make localization in the coal mine tunnels challenging. 


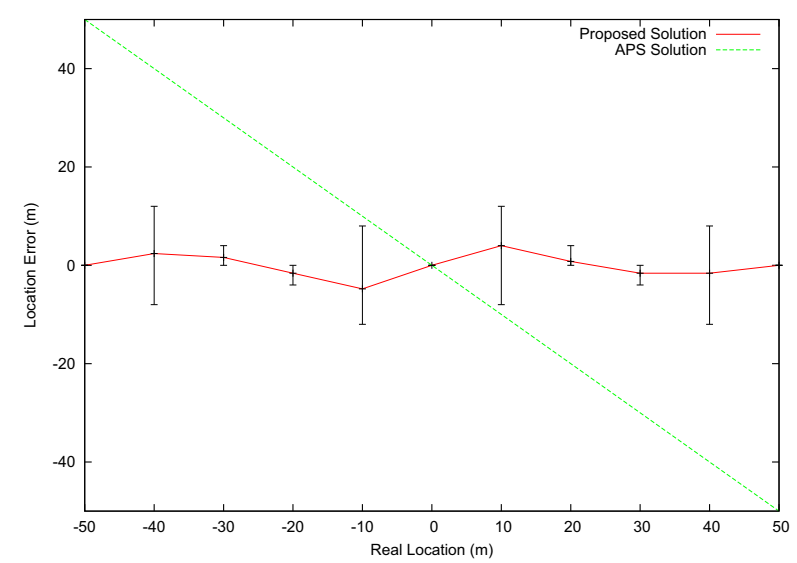

Fig. 7. Experiment results for the proposed solution.

In this paper, we proposed a practical localization solution for wireless sensor network deployed in linear topography. The proposed localization solution sets asymmetrical transmission range and makes best use of a priori location knowledge about the anchor nodes to obtain more reference information for localization of mobile nodes without any complex measurement, even if the distribution of mobile nodes is ultra sparse. The proposed solution is specially suitable for the linear topography, while for a common node distribution in a 2-dimension plane topography, it may become costly. It is because the density of anchor nodes needs to be large enough to cover the whole area, in case of having ultra sparse distribution of mobile nodes.

We also performed many experiments in a real coal mine tunnel. The experimental results of the coal mine tests show that the proposed localization solution can steadily achieve a high localization accuracy with an error no more than $13 \%$ of the transmission range of sensor nodes. While, under the same conditions, the existing localization ad-hoc positioning system solution has an error up to $44 \%$ of the transmission range.

\section{ACKNOWLEDGEMENT}

Authors would like to thank Bram Dil for his valuable comments and discussions. The work presented in this paper was partially supported by the NSF of China with Grant 60903171 and the National S\&T Major Project with Grant 2010ZX03006-002 and partially by the IST FP7 STREP GENESI: Green sEnsor NEtworks for structural monItoring.

\section{REFERENCES}

[1] H. L. B. H. Wellenhoff and J. Collins, Global positions system: theory and practice. Berlin, Germany: Springer Verlag, 1997.

[2] D. Niculescu and B. Nath, "Ad hoc positioning system using aoa," in Infocom, NJ, USA, 2003, pp. 1734-1743.

[3] G. B. J. Hightower and R. Want, "Spoton: an indoor 3d location sensing technology based on rf signal strength," CSE in University of Washington, Washington, Tech. Rep., Feb. 2000.

[4] C. H. A. Savvides and M. B. Strivastava, "Dynamic finegrained localization in ad-hoc networks of sensors," in Mobicom, Rome, Italy, 2001, pp. 166-179.
[5] D. Niculescu and B. Nath, "Ad hoc positioning system (aps)," in Proceedings of IEEE Global Telecommunication Conf. (GLOBECOM 01), San Antonio, TX, Nov. 2001, pp. 2926-2931.

[6] M. P. N. C. N. Patwari, A.O. Hero and R. ODea, "Relative location estimation in wireless sensor networks," IEEE Trans. Signal Process., vol. 51, no. 8, pp. 2137-2148, 2003.

[7] L. G. P. W. J. H. Vossiek, M. Wiebking and C. Heide, "Wireless local positioning," IEEE Microwave Magazine, vol. 4, no. 4, pp. 77-86, 2003.

[8] S. R. S.Y. Wong, J.G. Lim and W. Seah, "Density-aware hop-count localization in wireless sensor networks with variable density," in Proc. IEEE Wireless Communication and Networking Conf. (WCNC'05), New Orleans, LA USA, Mar. 2005, pp. 1848-1853.

[9] A. Nasipuri and K. Li, "A directionality based location discovery scheme for wireless sensor networks," in Proc. First ACM Int'l Workshop Wireless Sensor Networks and Applications (WSNA '02), Atlanta, Georgia, USA, Apr. 2002, pp. 105-111.

[10] B. B. J. S. T. He, C. Huang and T. Abdelzaher, "Range-free localization schemes for large scale sensor networks," in Mobicom, San Diego, California, USA, Sep. 2003.

[11] D. W. Y. Wang, X. Wang and D. Agrawal, "Localization algorithm using expected hop progress in wireless sensor networks," in Proceedings of Third IEEE Int'l Conf. Mobile Ad Hoc and Sensor Systems (MASS '06), Vancouver, BC, Canada, Oct. 2006, pp. 348-357. 
